\title{
Epidemiological Clinical Profile of Chronic Kidney Disease in Adults under 50 at the University Hospital of Brazzaville
}

\author{
Daniel Tony Eyeni Sinomono1*, Ginesse Ndinga-Okaka Sedan², Gael Honal Mahoungou1, \\ Eric Pierre Gandzali Ngabe ${ }^{3}$, Richard Moukengue Loumingou'1, Bertrand Ellenga-Mbolla", \\ Mohamed Arrayhani' ${ }^{5}$, Tarik Sqalli Houssain ${ }^{5}$
}

\author{
${ }^{1}$ Department of Nephrology and Dialysis, University Hospital of Brazzaville, Brazzaville, Congo \\ ${ }^{2}$ Hemodialysis Unit of the Medical Center for Specialists of the 15 years old plateau of Brazzaville, Brazzaville, Congo \\ ${ }^{3}$ Departement of Nephrology, Edith Lucie Bongo General Hospital of Oyo, Oyo, Congo \\ ${ }^{4}$ Department of Cardiology and Internal Medicine, University Hospital of Brazzaville, Brazzaville, Congo \\ ${ }^{5}$ Departement of Nephrology, University Hospital Hassan II of Fez, Fez, Morocco \\ Email: *eyetos1@gmail.com
}

How to cite this paper: Sinomono, D.T.E. Sedan, G.N.-O., Mahoungou, G.H., Ngabe, E.P.G., Loumingou, R.M., Ellenga-Mbolla, B., Arrayhani, M. and Houssain, T.S. (2021) Epidemiological Clinical Profile of Chronic Kidney Disease in Adults under 50 at the University Hospital of Brazzaville. Open Journal of Nephrology, 11, 358-369.

https://doi.org/10.4236/ojneph.2021.113029

Received: June 4, 2021

Accepted: August 16, 2021

Published: August 19, 2021

Copyright $\odot 2021$ by author(s) and Scientific Research Publishing Inc. This work is licensed under the Creative Commons Attribution-NonCommercial International License (CC BY-NC 4.0). http://creativecommons.org/licenses/by-nc/4.0/ (c) (i) \& Open Access

\begin{abstract}
Introduction: Chronic Kidney Disease (CKD) is a major global public health problem. In Congo, we have very few studies on it. Patients and Methods: We carried out an analytical cross-sectional study with retrospective data collection including patients aged 18 to 49 years, hospitalized for CKD in the nephrology department of University Hospital of Brazzaville, during the period from January 1, 2016 to August 31, 2020. The variables considered were socio-demographic, clinical, paraclinical, therapeutic and evolutionary. The statistical test used was Pearson's chi-square test. Results: CKD in those under 50 represented $21.1 \%$ of admissions and $35.6 \%$ of CKD. The mean age was 35.9 \pm 8.4 years. There was a male predominance with a sex ratio of 1.27 . Hypertension was the most common comorbidity (49.2\%), followed by HIV (13.6\%) and diabetes mellitus (13.2\%). Stage 5 CKD was most prevalent $(82.7 \%)$. Indeterminate kidney disease was in the order of $55.9 \%$. The low socioeconomic level (74.9\% of cases) hampered the achievement of dialysis in $66.3 \%$ of patients for whom this treatment was indicated. Failure to perform dialysis significantly influenced the occurrence of death ( $\mathrm{p}$-value $=0.005)$. Conclusion: $\mathrm{CKD}$ is common among people under 50 and more affects males with a high mortality rate due to lack of access to dialysis. The main causes are hypertension and HIV.
\end{abstract}

\section{Keywords}

Chronic Kidney Disease, Epidemiology, Brazzaville 


\section{Introduction}

Chronic Kidney Disease (CKD) represents a significant health challenge in the world population; in both developed and developing countries [1] [2] [3]. At the dialysis stage, it constitutes a real socio-health cost for national health systems. Its prevalence varies greatly from one region to another [2] [3] [4]. It has affected $10 \%-15 \%$ of the world's adult population [5] [6]. In Africa, this prevalence is between 30 and 186.5 per million population [7] [8].

Due to its progressive silent evolution, and its etiologies, dominated by arterial hypertension and diabetes mellitus; CKD is often mistakenly thought of as a disease of the elderly.

In Sub-Saharan Africa, where the population is mainly made up of young adults [8], the place of CKD among young Sub-Saharan adults remains imprecise due to the lack of scientific data.

In the Republic of Congo, specifically in Brazzaville, there is very little epidemiological data on the $\mathrm{CKD}$; those produced relate exclusively to chronic renal failure at all ages [9] [10]. A study is therefore necessary while placing particular emphasis on the under 50 age group. So we carried out our work with the objectives of: determining the frequency of $\mathrm{CKD}$ in adults under 50, describing the socio-demographic, clinical and biological characteristics; identify the etiologies and factors associated with death.

\section{Patients and Methods}

\subsection{Type, Place and Period of Study}

This was an analytical cross-sectional study, carried out in the nephrology and dialysis service of the University Hospital of Brazzaville (UHB). The data collection concerned patients hospitalized from January 1, 2016 to August 31, 2020, i.e. 4 years and 8 months.

\subsection{Study Population}

We included all patients aged 18 to 49 years, carriers of a CKD, defined independently of its cause by the presence for at least three consecutive months of a marker of renal damage and/or renal insufficiency, defined by a Glomerular Filtration Rate (GFR) less than $60 \mathrm{ml} / \mathrm{min} / 1.73 \mathrm{~m}^{2}$ [5]. Patients with acute kidney disease and those whose medical records were unusable were not included.

\subsection{Study Variables}

We studied the socio-demographic characteristics (age, sex, marital status, socioeconomic level). The socioeconomic level has been classified into three groups according to professional activities:

- Group 1: high socio-economic level. These include senior government officials or the private sector, import-export traders, army officers.

- Group 2: medium socio-economic level. It is made up of agents of the State 
or the private sector with a level of education equivalent to secondary school, shopkeepers, non-commissioned officers of the army.

- Group 3: low socio-economic level. It is made up of workers, laborers, farmers, soldiers and the unemployed.

We have also studied associated comorbidities; the etiologies of CKD; complications, stages based on the staging of KDIGO 2012 [2]; renal replacement techniques, mortality and associated factors.

\subsection{Collection of Data}

Data were collected from patient medical records and annual hospitalization registers. Anonymity has been retained.

The data was stored in Excel 2016 software. After the clearance and management of missing data, the analysis was performed in $\mathrm{R}$ studio software.

\subsection{Ethical Clearance}

To carry out our work, we obtained an authorization from the ethics committee of the faculty of health sciences of the Marien Ngouabi University and an authorization from the general management of the UHB.

\section{Results}

\subsection{Main Features}

During our study, 1789 patients were admitted to the nephrology and dialysis department of UHB. CKD was diagnosed in 1061 patients (or 59.3\% of admissions) including 378 adults under 50 years, is $21.13 \%$ of all admissions and $35.62 \%$ of all cases of CKD. Among those under 50; 135 had unusable files. Data could be collected from 243 patients. The socio-demographic characteristics of these patients are shown in Table 1.

\subsection{Co-Morbidities}

High blood pressure is the most common comorbidity at $49.17 \%$, followed by HIV (13.64\%) and diabetes mellitus (13.22\%). The distribution of patients according to comorbidities is presented in Table 2.

\subsection{CKD Stages}

The median serum creatinine was $1145 \mu \mathrm{mol} / \mathrm{L}$; the median GFR was $4.7 \mathrm{ml} / \mathrm{min}$ / $1.73 \mathrm{~m}^{2}(3.1 ; 9.6)$. Table 3 shows the distribution of patients according to the different stages of CKD.

\subsection{Lesion Diagnosis}

In the context of the lesion diagnosis, in 134 patients (55.14\%), the cause remained undetermined while in 109 patients (44.86\%), a lesion diagnosis could be determined. Table 4 shows the distribution of patients according to the lesion diagnosis. 
Table 1. Socio-demographic characteristics.

\begin{tabular}{ccc}
\hline Characteristics & & \\
\hline Average age & $36 \pm 8.4$ years & \\
Sex & Men & Women \\
Socioeconomic level & $136(56 \%)$ & $107(44 \%)$ \\
Low & & \\
Medium & $182(75.0 \%)$ & \\
High & $55(22.6 \%)$ & \\
& $6(02.4 \%)$ & \\
\hline
\end{tabular}

Table 2. Comorbidities associated with CKD.

\begin{tabular}{ccc}
\hline & $\mathbf{n}$ & $\%$ \\
\hline HBP & 119 & 49.0 \\
HIV & 52 & 21.4 \\
Diabetic sugar & 40 & 16.4 \\
Heart disease & 29 & 12.0 \\
Sickle cell anemia & 3 & 1.2 \\
Total & $\mathbf{2 4 3}$ & $\mathbf{1 0 0}$ \\
\hline
\end{tabular}

CKD: chronic kidney disease; HBP: high blood pressure; HIV: human immunodeficiency virus.

Table 3. Chronic kidney disease stages.

\begin{tabular}{ccc}
\hline & n & \% \\
\hline Stage 1 & 9 & 3.7 \\
Stage 2 & 4 & 1.6 \\
Stage 3A & 3 & 1.3 \\
Stage 3B & 8 & 3.3 \\
Stage 4 & 18 & 7.4 \\
Stage 5 & 201 & 82.7 \\
\hline
\end{tabular}

Table 4. Lesion diagnosis.

\begin{tabular}{cccc}
\hline & & $\mathbf{n}$ & $\%$ \\
\hline $\begin{array}{c}\text { Glomerular } \\
\text { nephropathies }\end{array}$ & $\bullet$ NGN & 47 & 29.6 \\
& $\bullet$ Glomerular proteinuria isolated & 13 & 5.3 \\
Tubulointerstitial nephropathies & 9 & 3.7 \\
Vascular nephropathies & 3 & 1.2 \\
\hline
\end{tabular}

CGN: chronic glomerulonephritis.

\subsection{Etiological Diagnosis}

Renal Biobsia Puncture (RBP) was indicated in 13 patients (5.35\%). Two patients 
did it overseas. While in the 11 other patients it was not done due to lack of technical platform. Histological analysis of the two RBP concluded with segmental and focal hyalinosis. The causes found as well as their proportion are listed in Table 5.

\subsection{Complications of CKD}

Anemia was the main complication of chronic renal failure found in $86 \%$ of patients. Apart from patients with stages 1 and 2 of CKD $(n=13)$, all other patients $(\mathrm{n}=230)$ had at least 2 complications related to chronic renal failure. Table 6 represents the proportions of each complication found.

\subsection{Renal Replacement Therapy}

Two hundred and one patients $(82.72 \%)$ had an indication for dialysis replacement therapy; only 51 of them (25.37\%) had done so.

Peritoneal dialysis was not performed during our study period.

No patient was transplanted.

\subsection{Mortality}

Death occurred in 66 patients $(27.2 \%)$ of which 59 were in stage $5(24.3 \%)$ and seven in stages 1, 2, 3 and 4 (2.9\%). Table 7 presents the different causes of death from CKD and Table 8 risk factors for death.

Table 5. Etiological diagnosis.

\begin{tabular}{ccc}
\hline & $\mathbf{n}$ & $\%$ \\
\hline Indeterminate & 134 & 55.1 \\
Hypertensive nephropathy & 38 & 15.6 \\
HIV-related nephropathy & 33 & 13.6 \\
Diabetic nephropathy & 32 & 13.2 \\
IgA Nephropathy & 3 & 1.2 \\
Primary segmental and focal hyalinosis & 2 & 0.8 \\
Autosomal dominant polycystic kidney disease & 1 & 0.4 \\
Total & 243 & 100 \\
\hline
\end{tabular}

IgA: Immunoglobin A.

Table 6. Complications of chronic kidney disease.

\begin{tabular}{ccc}
\hline Anemia & $\mathbf{n}$ & $\%$ \\
Hyperkalaemia & 209 & 86 \\
Hyper uricemia & 103 & 42.4 \\
Uremic encephalopathy & 18 & 8.6 \\
Acute pulmonary edema & 28 & 5.3 \\
Right femoral venous thrombosis & 15 & 0.8
\end{tabular}


Table 7. Different causes of death from chronic kidney disease.

\begin{tabular}{ccc}
\hline & $\mathbf{n}$ & $\%$ \\
\hline Severe acidosis & 20 & 30 \\
Uremic encephalopathy & 15 & 22.7 \\
Acute pulmonary edema & 10 & 15.6 \\
Ischemic heart disease & 5 & 7.6 \\
Severe anemia & 4 & 6 \\
Hemorrhagic stroke & 3 & 4.5 \\
Gastrointestinal bleeding & 2 & 3 \\
Coronavirus (COVID-19) & 2 & 3 \\
Indeterminate & 5 & 7.6 \\
Total & 66 & 100 \\
\hline
\end{tabular}

Table 8. Risk factors associated with death.

\begin{tabular}{|c|c|c|c|c|}
\hline Variables & $\begin{array}{c}\text { Total } \\
(n=243)\end{array}$ & $\begin{array}{l}\text { Living } \\
(\mathrm{n}=177)\end{array}$ & $\begin{array}{c}\text { Deceased } \\
(n=66)\end{array}$ & $\mathrm{p}$ \\
\hline \multicolumn{5}{|c|}{ Sexe } \\
\hline Women & 107 & 29 & 78 & \multirow{2}{*}{0.9} \\
\hline Men & 136 & 37 & 99 & \\
\hline \multicolumn{5}{|c|}{ Comorbidities } \\
\hline НBP & 119 & 87 & 32 & $<0.001$ \\
\hline DS & 40 & 25 & 15 & 0.3 \\
\hline HIV & 52 & 20 & 32 & $<0.001$ \\
\hline Heart Disease & 20 & 6 & 14 & $<0.001$ \\
\hline \multicolumn{5}{|c|}{ CKD stages } \\
\hline$S 1$ & 9 & 8 & 1 & \multirow{5}{*}{0.5} \\
\hline$S 2$ & 4 & 4 & 0 & \\
\hline$S 3$ & 11 & 10 & 1 & \\
\hline$S 4$ & 18 & 12 & 6 & \\
\hline$S 5$ & 201 & 143 & 58 & \\
\hline \multicolumn{5}{|c|}{ Complications } \\
\hline Hyperkalaemia & 103 & 70 & 33 & 0.15 \\
\hline APE & 18 & 8 & 10 & 0.02 \\
\hline Uremic encephalopathy & 25 & 7 & 18 & $<0.001$ \\
\hline Anemia & 209 & 143 & 66 & $<0.001$ \\
\hline
\end{tabular}

CKD: chronic kidney disease; HBP: high blood pressure; HIV: human immunodeficiency virus; APE: Acute pulmonary edema.

\section{Discussion}

Despite the youth of the Sub-Saharan African population, reported by the literature 
[8], we did not find any studies on CKD carried out exclusively in young adults under the age of 50. We compared our results with those of other studies on CKD at all ages but also with the interval of patients under 50 years if it was mentioned.

In our study, the CKD represented more than half of hospitalizations in the nephrology department of UHB (59.3\%); of which $35.6 \%$ were under 50 years old. CKD is therefore common in the latter. Our data join those of Ngoie S.M et al., in Lubumbashi, in 2015 who found a prevalence of 38.3\% of CKD among those under 50 years old in the only functional unit of nephrology and dialysis in their city [11].

However, our frequency, which is admittedly high, is lower than that found by Keylem et al., in 2015, in Ouagadougou (69.6\%) [12]. Sumaili et al. in Kinshasa, in 2009 , found a frequency of $89.1 \%$ in patients aged 20 to 59 years, of which $58.1 \%$ in the 20 to 39 year age group [13]. CKD is a reality among young people in Africa. The fact that our monocentric work took place in an intra-hospital ward could explain the frequency, admittedly high, but very far from that found by Sumaili et al. [13], where the data were multicentric. However, our data as well as those of Ngoie S.M in Lubumbashi remain far from those of Keylem in Ouagadougou, a single-center study carried out over a period of six months [12]. CKD among those under 50 in Brazzaville is less frequent compared to a country in West Africa. However, these results were inconsistent with those found in developed countries. In fact, in the United States and France, the prevalence of CKD was largely under-represented among those under 50 years of age with respective prevalence of $10.8 \%$ and $7.6 \%$ [3] [5]. This could be explained by the phenomenon of aging of the larger general population in developed countries, which is a consequence of improving health systems in the West.

We observed a male predominance (55.97\%). This predominance of the male sex in CKD is reported by several studies [9] [11] [12] [13]. These data are consistent with those of Sumaili EK. In Kinshasa [13], the high frequency of CKD in humans is explained by its rapid progression in this area [1] [3]. Our data conflict with that of Ahoui et al. in 2014 in Benin, which reported 51.26\% of women $(\mathrm{n}=651)$, i.e. a female/male sex ratio of 0.95 [4].

Socio-economic level is recognized as a key environmental social factor because it has implications for access to resources that help individuals take care of themselves and others. Few studies have examined the association of socioeconomic level with CKD. One hundred and eighty-two patients (74.9\%) had a low socioeconomic level compared to only six (2.4\%) who were found in the high social class. The privileged reach of this socio-economic group could be explained by their frequent use of prohibited drugs from the parallel market and herbal medicine. But, also by the lack of medical follow-up of chronic diseases such as hypertension, this lack of money. Cruz et al. [14] find the existence of an inverse correlation between CKD, low education and low income. Our results agree with those of Ramilitiana et al. in 2009, in Antananarivo, in Madagascar who mentioned $65.6 \%$ of patients with a low socioeconomic level [15]. However, they dif- 
fer from those of Houat Noria who reports in a study on the CKD in Algeria in 2014, 25\% for the high socioeconomic level, $70 \%$ for the average socioeconomic level and only $5 \%$ for the low socioeconomic level [16]. The specified fast age of our sample did not eliminate the predominance of the lower socioeconomic class in the CKD but rather accentuated it, since Sumaili in Kinshasa; Ramilitiana in Antananarivo have certainly found high frequencies of low socio-economic level but at values lower than ours [13] [15].

In our study a history of high blood pressure was found in $49.2 \%$ of patients. This high prevalence of hypertension is noted in all African studies [2] [4] [9] [13]; it is probable the result of changes in environmental and behavioral determinants such as the consumption of tobacco, alcohol, lack of physical activity, an inadequate diet and obesity as well as prolonged periods of exposure to these determinants, but also due to renal fibrosis responsible for hypereninism [5] [6]. The fight against CKD would begin by ensuring proper prevention of cardiovascular risk factors, especially hypertension, and their adequate management. Hypertension is an important cardiovascular risk factor and therefore an important renal risk factor. Indeed, it is the leading cause of CKD in Sub-Saharan Africa and is an important factor in the progression of CKD [2] [4] [13] [15]. However, by comparing our data with those in the literature, its frequency is lower in children under 50 than in patients with CKD in general since less than half of our patients were hypertensive; while other studies of all ages report more than half of hypertension in CKD [9] [12].

Diabetes was the second antecedent found in $18.6 \%$ of patients. This prevalence of diabetes varies greatly from country to country and is also influenced by dietary and behavioral factors.

The mean serum creatinine clearance was $13.7 \pm 26.2 \mathrm{ml} / \mathrm{min} / 1.73 \mathrm{~m}^{2}$. It was lower than that noted in Dakar by Ebata which was $19.95 \mathrm{ml} / \mathrm{min} / 1.73 \mathrm{~m}^{2}$ [17]. A prevalence of $97.67 \%$ of chronic kidney failure was noted in our study. This rate is similar to that found in Dakar (97.8\%) [17].

Due to its silent nature and the lack of awareness of kidney disease by the African population in general and by health personnel in particular, CKD is most often found at the end stage [9] [12] [13]. Thus all the African data reveal a high frequency of CKD at the terminal stage [12] [13]. At stage 5 of CKD, Sumaili et al. found a frequency of 78\%, Keylem et al. 75.3\% [12] [13]. These 2 studies are carried out at all ages. Our study limited to patients under 50 years old confirms this African trend since $82.72 \%$ of CKD are at the end stage. The young age of less than 50 years does not change this tendency of stage 5 predominance.

In stage I where the CKD may be asymptomatic with normal renal function; only $3.7 \%$ of patients $(\mathrm{n}=9)$ were found in our study. Sumaili et al. found $2 \%(\mathrm{n}$ = 7) [13]; Keylem found 1.5\% [12]. These data confirm the silent evolution of $\mathrm{CKD}$, as well as its ignorance and lack of routine health check-ups. The deficit of nephrologists, often forcing patients to travel long distances for a specialist consultation could also be responsible for the late diagnosis. In the West, where health coverage is almost complete and the number of nephrologists larger, studies show 
results totally opposite to ours [3] [5].

The causes of CKD are diverse. The search for these requires a deep anamnestic investigation. This etiological research is not only limited to the clinic but also largely involves biological examinations, particularly immunology and histology after performing a renal puncture biopsy (RPB); this is when the stage of fibrosis suspected clinically and on renal ultrasound is not reached. Renal histology occupies an important place in the etiological diagnosis of CKD [1] [3]. Indicated in 13 of our patients (5.3\%), the frequency of indications for RPB was low in our sample. Renal puncture biopsy has not been performed in more than $3 / 4$ of the patients who required it. These results could be explained by the weakness of our technical platform. In fact, the nephrology department of UHB does not have ultrasound machines or RPB guns. In addition, the many stains specific to renal histology and immunofluorescence are not available in our pathology laboratory. To the limits of the technical platform, we associate the high prevalence of stage 5 of CKD (55.1\%), a practical contraindication to performing a RPB. All these data reflect the high number of undetermined nephropathies (55.1\%). By comparing our data with those of the African literature, we also found a low frequency of performing RPB: Sumaili et al. in Kinshasa (DRC) performed a RPB en $13.1 \%$ of the patients collected in their study population [13]. In Togo, Amekoudi et al., found no RPB performed in their patients due to lack of technical platform [18].

Hypertensive nephropathy was the leading cause of CKD (15.6\%), followed by HIV-related nephropathy (13.6\%) and diabetic nephropathy (13.2\%). Hypertension has been described in the literature as the leading cause of CKD in black people [5] [14]. Sumaili et al. found a predominance of nephroangiosclerosis in the order of (26.9\%) [13]; Keylem et al. found 63.8\% [12]. The main cause of CKD in those under 50 is hypertension, contrasting with the study by DT Eyeni et al., carried out in the same department at all ages where the aetiologies were dominated by diabetes mellitus [9].

While hypertension and diabetes mellitus have been presented in all African studies as the top two causes of CKD at any age [4] [9] [13] [19]; our study limiting the age of patients to less than 50 years has particularly reversed this trend by putting HIV-related nephropathies in second place. Indeed, the HIV pandemic remains a topical issue with Africa as the most affected continent and young people as the most affected [20].

Segmental and focal hyalinosis was diagnosed in the two patients who had biopsy performed abroad. It is the primary cause of primary glomerulopathies in black subjects as reported by Segmental and focal hyalinosis, is reported by Sumaili et al. [13].

Treatment for CKD depends on the etiology. At stage 5, management is based on dialysis while waiting for a kidney transplant. The latter remains the treatment par excellence for stage 5 of CKD [21] [22]. In our study, the prevalence of stage 5 being high, there are therefore many indications for dialysis $(n=201$ or $82.7 \%)$. However, the rate of accessibility to dialysis was low (25.3\% of dialysis indica- 
tions). This poor accessibility is explained on the one hand by the absence of a public dialysis center in Brazzaville [9], and on the other hand by the high cost of a hemodialysis session in private centers [9] [13]. Our data join those of other African countries where access to dialysis is also difficult, particularly in Madagascar where only $1.2 \%$ of stage 5 patients have access to dialysis [15]. Kidney transplantation remains almost non-existent in Sub-Saharan Africa; with the exception of Abidjan, where the first kidney transplants in a country in Black Africa were performed in 2012 [23]. As in Kinshasa [13] and Ouagadougou [12], in our study renal transplantation was not performed in any patient.

The death rate from CKD was high in our study (27.1\%). The high prevalence of terminal CKD and the difficulties in accession replacement dialysis treatment could explain these results. Our data match those of: Keylem et al. in Ouagadougou (15 deaths or 21.7\%) [12].

The main cause of death found was severe acidosis, again reflecting the predominance of stage 5 in our sample.

Among the risk factors for death are: anemia, uremic encephalopathy, actute pulmonary edema reflecting severe acidosis ( $\mathrm{p}$-value $<0.05$ ). All these found risk factors for death are related to the severity of renal failure, thus reflecting delayed diagnosis of CKD but also difficult access to dialysis. Our results coincide with those of: Keylem et al. had found almost the same risk factors for death [12].

Otherwise, HIV infection also found as a risk factor for death; is considered a chronic systemic disease and can lead to many complications, including kidney damage. Since 2005, international companies (National Kidney Foundation) have recommended screening for kidney disease in all HIV-infected patients, the association of CRD with this is considered a factor of poor prognosis [24].

\section{Limits of the Study}

In assessing the extent of CKD in our study population, we were not spared from constraints. Indeed, the absence of certain medical files or certain key information made it impossible to use the data before 2016, thus justifying the small size of our sample. Also, the weakness of our technical platform was an obstacle to obtaining histological data, renal biopsies not being performed in our department during this study period. In addition, a prospective study would provide more details on the results.

\section{Conclusion}

The CKD of the young subject represents a concern in nephrology in Brazzaville because of its high frequency. The pathology concerns more the male sex and the patients consult the nephrology department late at stage 5 of the MRC. The predominant risk factors are represented by hypertension, HIV, diabetes mellitus and heart disease. Dialysis is indicated in the majority of cases but the rate of access to dialysis is low, due to the lack of a hemodialysis unit in public hospitals in Brazzaville, responsible for a high death rate. The prevention of cardiovascular risk 
factors and HIV is essential in our context, as it is the improvement of the technical platform.

\section{Contributions from Authors}

All the authors mentioned have made their mark on the completion of this article.

\section{Conflicts of Interest}

The author declares no conflict of interest.

\section{References}

[1] Jacquelinet, C. and Stengel, B. (2019) Chronic Kidney Disease. Elsevier, Amsterdam.

[2] Diallo, A.D., Niamkey, E. and Yao, B.B. (1997) Chronic Renal Failure in Côte d'Ivoire: Study of 800 Hospital Cases. Public Health, 90, 346-348.

[3] Coresh, J., Astor, B.C., Greene, T., et al. (2003) Prevalence of Chronic Kidney Disease and Decrease Kidney Function in the Adult US Population: Third National Health and Nutrition Examination Survey. American Journal of Kidney Diseases, 41, 1-12. https://doi.org/10.1053/ajkd.2003.50007

[4] Ahoui, S., Vigan, J., Albert, C., et al. (2016) Prevalence of Risk Factors for Chronic Kidney Disease in the Departments of Borgou and Alibori in Benin in 2014. Medicine and Public Health, 10, 1-12.

[5] HAS (2012) Guide to the Chronic Renal Disease Treatment Pathway in Adults 2012. https://www.has-sante.fr/jcms/c 1241102/fr/guide-parcours-de-soins-maladie-renal e-chronique-of-the-adult

[6] Rabiller, J. and Faure, S. (2019) From Chronic Kidney Disease to Kidney Transplantation. Pharmaceutical News, 584, 20-25. https://doi.org/10.1016/j.actpha.2019.01.014

[7] Pakasa, N.-M. and Sumaili, E.-K. (2012) Anatomopathological Peculiarities of Renal Disease in the Subject of Sub-Saharan Africa: Synthetic Review of Data from the Democratic Republic of the Congo. Annals of Diagnostic Pathology, 32, 40-52.

[8] Vimard, P. and Fassassi, R. (2012) Demography and Development in Africa: Retrospective and Prospective Elements. Cah Qué Demography, 40, 331-364. https://doi.org/10.7202/1011544ar

[9] Sinomono, D.T.E., Koumou, G.C. and Loumingou, R. (2017) Epidemiological Profile of Chronic Renal Failure at Brazzaville University Hospital in 2016. Therapeutic Nephrology, 13, 396. https://doi.org/10.1016/j.nephro.2017.08.302

[10] Missamou, A., Sinomono, D.T.E., Loumingou, R., et al. (2019) Management of Renal Failure at the Pointe-Noire General Hospital in Congo: Between Realities and Hopes. Therapeutic Nephrology, 15, 344. https://doi.org/10.1016/j.nephro.2019.07.197

[11] Ngoie, S.M., Mulenga, P., Mukuku, O., et al. (2017) Chronic Renal Disease: Associated Factors, Etiologies, Clinical and Biological Characteristics in Lubumbashi in the Democratic Republic of Congo. The Pan African Medical Journal, 28, 1-11.

[12] Keylem, C.G., Sempore, Y.W., Yameogo, A.A., et al. (2020) Diagnosis and Management of Chronic Kidney Disease in a Context of Limited Resources: Specificities and Difficulties of an Internal Medicine Department. African Journal of Internal Medicine, 7, 11-19.

[13] Sumaili, E.K., Krzesinski, J.-M., Cohen, E.P., et al. (2010) Epidemiology of Chronic 
Kidney Disease in the Democratic Republic of the Congo: A Synthetic Review of Studies from Kinshasa, the Capital. Therapeutic Nephrology, 6, 232-239.

[14] Cruz, I.A. and Hosten, A.O. (1989) An Update of the End-Stage Renal Disease Program at Howard University Hospital. Transplantation Proceedings, 21, 3892-3894.

[15] Ramilitiana, B., Ranivoharisoa, E.M., Dodo, M., et al. (2016) A Retrospective Study on the Incidence of Chronic Renal Failure in the Department of Internal Medicine and Nephrology of the University Hospital of Antananarivo. The Pan African Medical Journal, 23, 89-96. https://doi.org/10.11604/pamj.2016.23.141.8874

[16] Houat, N., et al. (2017) Incidence of End-Stage Chronic Renal Failure in Maghnia. Santé Maghreb, 12, 8-14.

[17] Seck, S., Ka, F. and Cisse, M. (2014) Prevalence Survey of Chronic Kidney Disease in the Northern Region of Senegal. Therapeutic Nephrology, 10, 399. https://doi.org/10.1016/j.nephro.2014.07.128

[18] Amekoudi, E.Y., Sabi, K.A., Noto-Kadou-Kaza, B. and Combe, C. (2016) Epidemiology of Chronic Kidney Disease in Nephrology Consultation at the Sylvanus-Olympio CHU in Togo. Nephrology \& Therapeutics, 12, 400. https://doi.org/10.1016/j.nephro.2016.07.113

[19] Tiendrebéogo, O. (2020) Epidemiology of Chronic Nephropathies in the Internal Medicine Department of the Yalgado Ouédraogo University Hospital Center (CHUYO) in Ouagadougou. African Journal of Internal Medicine, 7, 11-19.

[20] UNAIDS, WHO and UNDP (2011) HIV/AIDS in Sub-Saharan Africa: An Update on the Epidemic and Health Sector Progress towards Universal Access. Situation Report.

https://www.unaids.org/sites/default/files/media asset/20111130 UA Report en 1.pdf

[21] National Kidney Foundation-Kidney Disease Outcomes Quality Initiative (NKFKDOQI) (2002) Clinical Practice Guidelines for Chronic Kidney Disease: Evaluation, Classification, and Stratification. Journal of American Kidney Disease, 39, 29-40.

[22] El Nahas, M., Kawar, B.S. and El Kossi, M. (2012) Epidemiology, Etiology, Pathophysiology and Staging of Chronic Kidney Diseases. In: Edgar, V.L. and Allen, R.N., Eds., Nephrology Secrets, 3rd Edition, Elsevier, Amsterdam, 131-141. https://doi.org/10.1016/B978-1-4160-3362-2.00028-2

[23] Coulibaly, N., Ackoundou-N'guessan, C., Nguessan, Y.F., Aye, Y., Guei, M.C., Toure, D.J.J., Hoang, A.D. and Sangare, I. (2016) Surgical Aspects and Preliminary Results of Renal Transplantation in Côte d'Ivoire. Uro' Andro, 1, 256-260.

[24] Izzedine, H. (2009) Renal Diseases during HIV Infection. Letter of Infectious Diseases, 24, 146-156. 